\title{
Asymptotic Markov inequality on Jordan arcs*
}

\author{
Vilmos Totik ${ }^{\dagger}$
}

November 16, 2016

\begin{abstract}
Markov's inequality for the derivative of algebraic polynomials is considered on $C^{2}$ smooth Jordan arcs. The asymptotically best estimate is given for the $k$-th derivative for all $k=1,2, \ldots$ The best constant is related to the behavior around the endpoints of the arc of the normal derivative of the Green's function of the complementary domain. The result is deduced from the asymptotically sharp Bernstein inequality for the $k$-th derivative at inner points of a Jordan arc, which is derived from a recent result of S. I. Kalmykov and B. Nagy on the Bernstein inequality on analytic arcs. In the course of the proof we shall also need to reduce the analyticity condition in this last result to $C^{2}$ smoothness.
\end{abstract}

\section{Introduction and results}

The Bernstein inequality

$$
\left|P_{n}^{\prime}(x)\right| \leq \frac{n}{\sqrt{1-x^{2}}}\left\|P_{n}\right\|_{[-1,1]}, \quad x \in(-1,1),
$$

and the Markov inequality

$$
\left\|P_{n}^{\prime}\right\|_{[-1,1]} \leq n^{2}\left\|P_{n}\right\|_{[-1,1]}
$$

are arguably the most important polynomial inequalities that have lots of applications (see e.g. [2], [4, Corollary 4.1.2] and [8], [4, Theorem 4.1.4] for these inequalities and [4] for some classical applications). In (1.1) and (1.2) the norm $\|\cdot\|_{K}$ is the supremum norm on $K$, and $P_{n}$ denotes an arbitrary (complex) algebraic polynomial of degree at most $n$. The Bernstein inequality (1.1) gives a better estimate for $\left|P_{n}^{\prime}(x)\right|$ if $x$ is not too close to the endpoints \pm 1 , but close to the endpoints (1.2) is better. Both inequalities are sharp, and they have many variants and generalizations.

Markov-type inequalities for general continua (connected compact sets) have been given, among others, by C. Pommerenke [14] and A. Eremenko [5]. In the

* AMS Classification 42A05. Keywords: Markov inequality, Jordan arcs, normal derivatives of Green's functions

†Supported by NSF DMS 1564541 
recent work [7] S. Kalmykov and B. Nagy found the precise asymptotic analogue of the Bernstein inequality on analytic Jordan arcs. The aim of this paper is to remove the analyticity requirement (replace it by $C^{2}$ smoothness), and to prove also the analogue of the Markov inequality on such arcs.

Let $\Gamma$ be a Jordan arc (homeomorphic image of a segment), and let us denote by $g_{\overline{\mathbf{C}} \backslash \Gamma}(z, \infty)$ the Green's functions of the complement $\overline{\mathbf{C}} \backslash \Gamma$ of $\Gamma$ with pole at infinity (see e.g. the books [1], [15] or [18] for the concepts we use from potential theory). For simplicity we shall often write $g_{\overline{\mathbf{C}} \backslash \Gamma}(z)$ instead of $g_{\overline{\mathbf{C} \backslash \Gamma}}(z, \infty)$. We say that $\Gamma$ is $C^{2}$ smooth if it has a twice continuously differentiable parametrization $\gamma(t), t \in[-1,1]$, such that $\gamma^{\prime}(t) \neq 0$. In a similar manner, we say that $\Gamma$ is analytic if it has a parametrization $\gamma(t), t \in[-1,1]$, such that $\gamma(t)$ can be expanded into a power series around each point $t_{0} \in[-1,1]$, and $\gamma^{\prime}(t) \neq 0$ for any $t \in[-1,1]$.

If $\mathbf{n}_{ \pm}$denote the unit normal vectors to $\Gamma$ on the two sides of $\Gamma$, then the Kalmykov-Nagy theorem [7] is the following: if $\Gamma$ is analytic, then

$$
\left|P_{n}^{\prime}\left(z_{0}\right)\right| \leq(1+o(1)) n\left\|P_{n}\right\|_{\Gamma} \max \left(\frac{\partial g_{\overline{\mathbf{C} \backslash \Gamma}}\left(z_{0}\right)}{\partial \mathbf{n}_{+}}, \frac{\partial g_{\overline{\mathbf{C} \backslash \Gamma}}\left(z_{0}\right)}{\partial \mathbf{n}_{-}}\right)
$$

for any $z_{0} \in \Gamma$ different from the endpoints of $\Gamma$. Here $o(1)$ tends to 0 uniformly in $P_{n}$ as $n$, the degree of $P_{n}$, tends to infinity. It also follows from [7] that the inequality holds uniformly in $z_{0} \in \Gamma$ provided $z_{0}$ stays away from the endpoints of $\Gamma$. (1.3) solved a problem raised in [11].

The estimate (1.3) is best possible: for every $z_{0} \in \Gamma$ that is different from the endpoints there are nonzero polynomials for which ${ }^{1}$

$$
\left|P_{n}^{\prime}\left(z_{0}\right)\right| \geq(1+o(1)) n\left\|P_{n}\right\|_{\Gamma} \max \left(\frac{\partial g_{\overline{\mathbf{C} \backslash \Gamma}}\left(z_{0}\right)}{\partial \mathbf{n}_{+}}, \frac{\partial g_{\overline{\mathbf{C}} \backslash \Gamma}\left(z_{0}\right)}{\partial \mathbf{n}_{-}}\right),
$$

see [7], [11].

Our first result is that (1.3) is true on $C^{2}$ Jordan arcs.

Theorem 1 Let $\Gamma$ be a $C^{2}$-smooth Jordan arc on the plane and let $z_{0} \in \Gamma$ be a point that is different from the endpoints of $\Gamma$. Then (1.3) is true.

Furthermore, if $J$ is a closed subarc of $\Gamma$ that does not contain either of the endpoints of $\Gamma$, then (1.3) holds uniformly $z_{0} \in J$.

It follows from the proof that if one wants to prove the theorem at a single point $z_{0}$, then all one needs for $\Gamma$ is $C^{2}$ smoothness in a neighborhood of $z_{0}$.

Theorem 1 will be deduced from its version given in [7] for analytic arcs.

To state the corresponding Markov inequality let $A, B$ be the endpoints of Г. Define

$$
\Omega_{ \pm}(A)=\lim _{z \rightarrow A, z \in \Gamma} \sqrt{|z-A|} \frac{\partial g_{\overline{\mathbf{C}} \backslash \Gamma}(z, \infty)}{\partial \mathbf{n}_{ \pm}(z)},
$$

${ }^{1}$ We use the standard convention that $o(1)$ is a quantity, not necessarily positive, that tends to 0 . In particular, $A_{n} \geq\left(1+o(1) B_{n}\right.$ may not imply that $A_{n} \geq B_{n}$ for large $n$. 
It will turn out that these limits exist and $\Omega_{+}(A)=\Omega_{-}(A)$, so let $\Omega(A)=\Omega_{\Gamma}(A)$ be the common value. Define $\Omega(B)$ similarly.

For the interval $[-1,1]$ the two endpoints \pm 1 play symmetric role. This is no longer true for Jordan arcs, so one should speak about separate Markov inequalities about $A$ and $B$. Let $U$ be any fixed closed neighborhood of $A$ that does not contain $B$. Then the Markov inequality about the point $A$ takes the form

$$
\left\|P_{n}^{\prime}\right\|_{U \cap \Gamma} \leq(1+o(1)) n^{2} 2 \Omega(A)^{2}\left\|P_{n}\right\|_{\Gamma},
$$

and this is sharp (see Theorems 2 and 3 below). In particular, the global Markov inequality for the whole arc is

$$
\left\|P_{n}^{\prime}\right\|_{\Gamma} \leq(1+o(1)) n^{2} 2 \max (\Omega(A), \Omega(B))^{2}\left\|P_{n}\right\|_{\Gamma},
$$

and this is sharp again regarding the constant on the right. However, if we iterate this to obtain an estimate for higher derivatives, then we get that for every fixed $k=1,2, \ldots$ we have

$$
\left\|P_{n}^{(k)}\right\|_{\Gamma} \leq(1+o(1)) n^{2 k} 2^{k} \max (\Omega(A), \Omega(B))^{2 k}\left\|P_{n}\right\|_{\Gamma},
$$

which is not sharp. So to obtain sharp bounds we shall have to deal with the $k$-th derivative from the outset. This is given in the next theorem.

As a guide, consider the situation on $[-1,1]$. If we iterate Markov's inequality (1.2), then we get for the $k$-th derivative of a polynomial

$$
\left\|P_{n}^{(k)}\right\|_{[-1,1]} \leq n^{2}(n-1)^{2} \cdots(n-k+1)^{2}\left\|P_{n}\right\|_{[-1,1]},
$$

but the exact result is the general Markov inequality (see [9] or [10, Theorem 1.2.2, Sec. 6.1.2]),

$$
\left\|P_{n}^{(k)}\right\|_{[-1,1]} \leq \frac{n^{2}\left(n^{2}-1^{2}\right)\left(n^{2}-2^{2}\right) \cdots\left(n^{2}-(k-1)^{2}\right)}{(2 k-1) ! !}\left\|P_{n}\right\|_{[-1,1]},
$$

(recall that $(2 k-1) ! !=(2 k-1)(2 k-3) \cdots 3 \cdot 1)$, which was proven by $\mathrm{V}$. A. Markov, the younger brother of A. A. Markov who found (1.2).

The exact form of (1.5) on general arcs is out of reach (namely to get the precise constant for every $n$ ), however if we write (1.5) in the asymptotic form

$$
\left\|P_{n}^{(k)}\right\|_{[-1,1]} \leq(1+o(1)) \frac{n^{2 k}}{(2 k-1) ! !}\left\|P_{n}\right\|_{[-1,1]},
$$

then we can recapture this asymptotic form for general arcs.

Theorem 2 Let $\Gamma$ be a $C^{2}$ Jordan arc with endpoints $A, B$, and let $k$ be a positive integer. Let further $U$ be a closed neighborhood of $A$ that does not contain $B$. Then, for polynomials $P_{n}$ of degree at most $n=1,2, \ldots$, we have

$$
\left\|P_{n}^{(k)}\right\|_{U \cap \Gamma} \leq(1+o(1)) n^{2 k} \frac{2^{k} \Omega(A)^{2 k}}{(2 k-1) ! !}\left\|P_{n}\right\|_{\Gamma},
$$

where $o(1) \rightarrow 0$ as $n \rightarrow \infty$ and this o(1) is independent of $P_{n}$. 

(1.6).

If $\Gamma=[-1,1]$, then $\Omega(-1)=\Omega(1)=1 / \sqrt{2}$, so in this special case we obtain

The next theorem shows that the constant $2^{k} \Omega(A)^{2 k} /(2 k-1) ! !$ is asymptotically the best possible in Theorem 2 .

Theorem 3 For every $\Gamma$ there are polynomials $P_{n} \not \equiv 0$, of degree $n=1,2, \ldots$ such that

$$
\left|P_{n}^{(k)}(A)\right| \geq(1+o(1)) n^{2 k} \frac{2^{k} \Omega(A)^{2 k}}{(2 k-1) ! !}\left\|P_{n}\right\|_{\Gamma} .
$$

From here the asymptotically sharp global Markov inequality

$$
\left\|P_{n}^{(k)}\right\|_{\Gamma} \leq(1+o(1)) n^{2 k} \frac{2^{k} \max (\Omega(A), \Omega(B))^{2 k}}{(2 k-1) ! !}\left\|P_{n}\right\|_{\Gamma},
$$

is an immediate consequence. As an example consider the circular arc $\Gamma=$ $\left\{e^{i t} \mid-\alpha \leq t \leq \alpha\right\}$ for some $\alpha \in(0, \pi)$. Since the two normal derivatives of the Green's function $g_{\overline{\mathbf{C} \backslash \Gamma}}$ at the point $e^{i \theta} \in \Gamma$ are (see [11, Proposition 3])

$$
\frac{\partial g_{\overline{\mathbf{C} \backslash \Gamma}}\left(e^{i \theta}\right)}{\partial \mathbf{n}_{ \pm}}=\frac{1}{2}\left( \pm 1+\frac{\sqrt{2} \cos (\theta / 2)}{\sqrt{\cos \theta-\cos \alpha}}\right)
$$

with appropriate choice of $\mathbf{n}_{ \pm}$, we obtain that for both endpoints

$$
\Omega(A)=\Omega(B)=\frac{1}{2} \sqrt{\cot \frac{\alpha}{2}}
$$

and hence

$$
\left\|P_{n}^{(k)}\right\|_{\Gamma} \leq(1+o(1)) n^{2 k} \frac{(\cot (\alpha / 2))^{k}}{2^{k}(2 k-1) ! !}\left\|P_{n}\right\|_{\Gamma} .
$$

The $k=1$ case of this inequality is essentially due to V. S. Videnskii [19] (see also [3, p. 243]) who proved Markov-type inequalities for trigonometric polynomials on intervals shorter than $2 \pi$. (1.8) is the best possible, since for some polynomials we have

$$
\left\|P_{n}^{(k)}\right\|_{\Gamma} \geq(1+o(1)) n^{2 k} \frac{(\cot (\alpha / 2))^{k}}{2^{k}(2 k-1) ! !}\left\|P_{n}\right\|_{\Gamma} .
$$
1 :

In proving Theorem 2 we shall need the higher derivative version of Theorem

Theorem 4 Let $\Gamma$ be a $C^{2}$-smooth Jordan arc on the plane and let $J$ be a subarc of $\Gamma$ that has no common endpoint with $\Gamma$. Then, for any fixed $k \geq 1$ and for all polynomials $P_{n}$ of degree at most $n=1,2, \ldots$, we have

$$
\left|P_{n}^{(k)}\left(z_{0}\right)\right| \leq(1+o(1)) n^{k}\left\|P_{n}\right\|_{\Gamma} \max \left(\frac{\partial g_{\overline{\mathbf{C} \backslash \Gamma}}\left(z_{0}\right)}{\partial \boldsymbol{n}_{+}}, \frac{\partial g_{\overline{\mathbf{C} \backslash \Gamma}}\left(z_{0}\right)}{\partial \boldsymbol{n}_{-}}\right)^{k}
$$

uniformly in $z_{0} \in J$ as $n \rightarrow \infty$.

This is again sharp for every $k$ and every $z_{0}$ different from the endpoints of $\Gamma$ (see the proof of Theorem 3). 


\section{Proof of Theorem 1}

In the proof we shall frequently identify a Jordan arc or curve with its parametric representation.

By assumption, $\Gamma$ has a twice differentiable parametrization $\gamma(t), t \in[-1,1]$, such that $\gamma^{\prime}(t) \neq 0$ and $\gamma^{\prime \prime}$ is continuous. We may assume that $z_{0}=0$ and that the real line is tangent to $\Gamma$ at 0 . By reparametrization we may then assume that $\gamma(0)=0, \gamma^{\prime}(0)>0$. There is an $M_{0}$ such that for all $t \in[-1,1]$ we have

$$
\frac{1}{M_{0}} \leq\left|\gamma^{\prime}(t)\right| \leq M_{0}, \quad\left|\gamma^{\prime \prime}(t)\right| \leq M_{0}
$$

Set $\gamma_{0}=\gamma=\Gamma$, and for some $0<\tau_{0}<1$ and for all $0<\tau \leq \tau_{0}$ we are going to construct a family of analytic Jordan $\operatorname{arcs} \gamma_{\tau}$ with similar properties as $\gamma$. Indeed, for $\tau>0$ choose a polynomial $g_{\tau}$ such that

$$
\left|\gamma^{\prime \prime}-g_{\tau}\right| \leq \tau,
$$

and set

$$
\gamma_{\tau}(t)=\int_{0}^{t}\left(\int_{0}^{u} g_{\tau}(\xi) d \xi+\gamma_{0}^{\prime}(0)\right) d u, \quad t \in[-1,1] .
$$

It is clear that for these $\gamma_{\tau}^{\prime}(0)=\gamma_{0}^{\prime}(0)=\gamma^{\prime}(0)$ and

$$
\left|\gamma_{\tau}(t)-\gamma_{0}(t)\right| \leq \tau|t|^{2}, \quad\left|\gamma_{\tau}^{\prime}(t)-\gamma_{0}^{\prime}(t)\right| \leq \tau|t|, \quad t \in[-1,1] .
$$

In view of $(2.1)$ and $\gamma^{\prime}(0)>0$ we see that ${ }^{2}$

$$
\Re \gamma_{0}^{\prime}(t) \geq 1 / 2 M_{0}, \quad|t| \leq 1 / 2 M_{0}^{2},
$$

and then we obtain from (2.4) that if $|t| \leq 1 / 4 M_{0}^{2}$, then $\Re \gamma_{\tau}^{\prime}(t) \geq 1 / 4 M_{0}$. Therefore, for $\left|t_{1}\right|,\left|t_{2}\right| \leq 1 / 4 M_{0}^{2}$ we have

$$
\left|\gamma_{\tau}\left(t_{1}\right)-\gamma_{\tau}\left(t_{2}\right)\right| \geq\left|t_{1}-t_{2}\right| / 4 M_{0}
$$

and similarly follows that, in general, if $\left|t_{2}-t_{1}\right| \leq 1 / 4 M_{0}^{2}$, then (2.6) is true (just use the vector $\gamma^{\prime}\left(t_{1}\right)$ in the preceding argument instead of $\left.\gamma^{\prime}(0)\right)$. On the other hand, since $\gamma_{0}$ is a Jordan arc, there is an $M_{1}$ such that for $\left|t_{2}-t_{1}\right| \geq 1 / 4 M_{0}^{2}$ we have $\left|\gamma_{0}\left(t_{1}\right)-\gamma_{0}\left(t_{2}\right)\right| \geq 1 / M_{1}$ with some $M_{1}$, hence if $\tau_{0}<1 / 4 M_{1}$ and $\tau \leq \tau_{0}$, then we have $\left|\gamma_{\tau}\left(t_{1}\right)-\gamma_{\tau}\left(t_{2}\right)\right| \geq 1 / 2 M_{1}$.

Thus, for all $0<\tau \leq \tau_{0}$ the $\gamma_{\tau}$ is an analytic Jordan arc which passes through the origin, and which has the real line as its tangent at 0 .

Next, we use that, by [17, Theorem 7.1], $g_{\overline{\mathbf{C} \backslash \gamma_{\tau}}}(z)$ are uniformly Hölder $1 / 2$ continuous:

$$
g_{\overline{\mathbf{C} \backslash \gamma_{\tau}}}(z) \leq M_{2} \operatorname{dist}\left(z, \gamma_{\tau}\right)^{1 / 2}
$$

with a constant $M_{2}$ that depends only on the diameter of $\gamma_{\tau}$, and hence independent of $\tau$. In particular, this implies that $\left\{g_{\overline{\mathbf{C}} \backslash \gamma_{\tau}}(z) \mid 0 \leq \tau \leq \tau_{0}\right\}$ are

\footnotetext{
${ }^{2}$ Here, and in what follows, $A / B C$ means $A /(B C)$
} 
uniformly equicontinuous on all $\mathbf{C}$ and uniformly bounded on compact subsets of C. Thus, it follows from (2.4) that

$$
g_{\overline{\mathbf{C} \backslash \gamma_{0}}}(z) \leq M_{2} \sqrt{\tau}, \quad z \in \gamma_{\tau} \quad \text { and } \quad g_{\overline{\mathbf{C} \backslash \gamma_{\tau}}}(z) \leq M_{2} \sqrt{\tau}, \quad z \in \gamma_{0} .
$$

We have a better estimate around the origin, namely it will be proven in Appendix 1 that the Green's functions $g_{\overline{\mathbf{C}} \backslash \gamma_{\tau}}, 0 \leq \tau \leq \tau_{0}$, are uniformly Hölder 1 continuous in a neighborhood of the origin which contains the $\operatorname{arcs}\left\{\gamma_{\tau}(t)\right.$, $t \in[-1 / 2,1 / 2]\}$. In view of (2.4) this implies that for $t \in[-1 / 2,1 / 2]$

$$
g_{\overline{\mathbf{C} \backslash \gamma_{0}}}(z) \leq M_{3} \tau t^{2}, \quad z=\gamma_{\tau}(t) \quad \text { and } \quad g_{\overline{\mathbf{C} \backslash \gamma_{\tau}}}(z) \leq M_{3} \tau t^{2}, \quad z=\gamma_{0}(t)
$$

with some $M_{3}$ independent of $\tau \leq \tau_{0}$.

(2.8) and (2.9) can be written as the global estimate

$$
g_{\overline{\mathbf{C} \backslash \gamma_{0}}}(z) \leq M_{4} \sqrt{\tau}|z|^{2}, \quad z \in \gamma_{\tau} \quad \text { and } \quad g_{\overline{\mathbf{C} \backslash \gamma_{\tau}}}(z) \leq M_{4} \sqrt{\tau}|z|^{2}, \quad z \in \gamma_{0} \text {, }
$$

with some constant $M_{4}$, where it is also used that, by (2.6), we have for $z=\gamma_{\tau}(t)$ the inequality $|z| \geq|t| / \bar{M}_{4}$ with some $\bar{M}_{4}$ that is independent of $\tau$ and $t$.

It is also proven in Appendix 1 that no matter how $\eta>0$ is given, there is a $\tau_{\eta}<\tau_{0}$ such that for $\tau<\tau_{\eta}$ we have

$$
\frac{\partial g_{\overline{\mathbf{C} \backslash \gamma_{\tau}}}(0)}{\partial \mathbf{n}_{ \pm}}<(1+\eta) \frac{\partial g_{\overline{\mathbf{C} \backslash \gamma_{0}}}(0)}{\partial \mathbf{n}_{ \pm}},
$$

where $\mathbf{n}_{ \pm}$denote the two (common) normals to $\gamma_{\tau}, 0 \leq \tau \leq \tau_{0}$, at the origin. In fact, it is proven in (6.1) that $\partial g_{\overline{\mathbf{C} \backslash \gamma_{\tau}}}(0) / \partial \mathbf{n}_{ \pm} \rightarrow \partial g_{\overline{\mathbf{C} \backslash \gamma_{0}}}(0) / \partial \mathbf{n}_{ \pm}$as $\tau \rightarrow 0$, and since the latter normal derivatives are not zero (see Remark 7 in Section $6)$, the inequality (2.11) follows.

The estimates given for the Green's functions have the following use. In view of the Bernstein-Walsh lemma [20, p. 77], (2.10) yields that if $P_{n}$ is a polynomial of degree at most $n$, then for $z \in \gamma_{\tau}$ we have

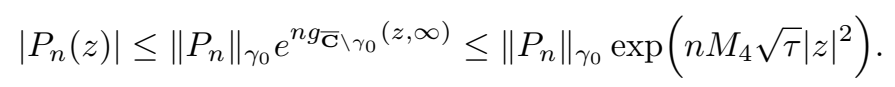

Consider the closure of the set $\cup_{0 \leq \tau \leq \tau_{0}} \gamma_{\tau}$ and its polynomial convex hull

$$
K=\operatorname{Pc}\left(\overline{\bigcup_{0 \leq \tau \leq \tau_{0}} \gamma_{\tau}}\right),
$$

which is the union of that closure with all the bounded components of its complement. Since all $\gamma_{\tau}$ pass through the origin where all of them have the real line as their tangent, and since all of them have uniformly bounded curvatures by their uniform $C^{2}$ property, it follows that there is a disk (say in the upper half plane) in the complement of $K$ which contains the point 0 on its boundary (in fact, the circle $C_{a}$ about $i a$ with radius $a$ suffices for small $a>0$ ). But then (see [17, Theorem 4.1]) there are constants $c_{0}, C_{0}$ and for each $m$ polynomials $Q_{m}$ of degree at most $m$ such that 
(i) $Q_{m}(0)=1$,

(ii) $\left|Q_{m}(z)\right| \leq 1, \quad z \in K$,

(iii) $\left|Q_{m}(z)\right| \leq C_{0} e^{-c_{0} m|z|^{2}}, \quad z \in K$.

After these preparations let $P_{n}$ be a polynomial of degree at most $n$, and for some small $\varepsilon>0$ consider $P_{n}(z) Q_{\varepsilon n}(z)$. We estimate this polynomial on $\gamma_{\tau}$ as follows. Let $z \in \gamma_{\tau}$ and let $0<\eta<1$ be given.

Case I. If $|z| \leq \sqrt{2 \log C_{0} / c_{0} \varepsilon n}$, then (2.12) and (ii) yield

$$
\left|P_{n}(z) Q_{\varepsilon n}(z)\right| \leq \exp \left(M_{4} \sqrt{\tau} 2 \log C_{0} / c_{0} \varepsilon\right)\left\|P_{n}\right\|_{\gamma_{0}},
$$

and the right hand side is smaller than $(1+\eta)\left\|P_{n}\right\|_{\gamma_{0}}$ if $\tau<\left(\eta c_{0} \varepsilon / 4 M_{4} \log C_{0}\right)^{2}$.

Case II. If $|z|>\sqrt{2 \log C_{0} / c_{0} \varepsilon n}$, then (2.12) and (iii) yield

$$
\left|P_{n}(z) Q_{\varepsilon n}(z)\right| \leq\left\|P_{n}\right\|_{\gamma_{0}} C_{0} \exp \left(n M_{4} \sqrt{\tau}|z|^{2}-c_{0} \varepsilon n|z|^{2}\right) .
$$

For $\sqrt{\tau}<c_{0} \varepsilon / 2 M_{4}$ the exponent is at most

$$
-n\left(c_{0} / 2\right) \varepsilon|z|^{2} \leq \log \left(1 / C_{0}\right)
$$

so in this case we have

$$
\left|P_{n}(z) Q_{\varepsilon n}(z)\right| \leq\left\|P_{n}\right\|_{\gamma_{0}} .
$$

So far we have shown that

$$
\left\|P_{n} Q_{\varepsilon n}\right\|_{\gamma_{\tau}} \leq(1+\eta)\left\|P_{n}\right\|_{\gamma_{0}}
$$

if $\tau$ is small, say $\tau<\tau_{\eta}^{*}$. Fix such a $\tau$.

The polynomial $P_{n} Q_{\varepsilon n}$ has degree at most $n(1+\varepsilon)$, so by the KalmykovNagy estimate (1.3) applied to $\gamma_{\tau}$ (note that this is an analytic Jordan arc) we have for large $n$

$$
\left|\left(P_{n} Q_{\varepsilon n}\right)^{\prime}(0)\right| \leq(1+o(1)) n(1+\varepsilon)\left\|P_{n} Q_{\varepsilon n}\right\|_{\gamma_{\tau}} \max \left(\frac{\partial g_{\overline{\mathbf{C}} \backslash \gamma_{\tau}}(0)}{\partial \mathbf{n}_{+}}, \frac{\partial g_{\overline{\mathbf{C}} \backslash \gamma_{\tau}}(0)}{\partial \mathbf{n}_{-}}\right) .
$$

On the right hand side we can use (2.16), as well as the fact that, in view of (2.11), the last factor is at most

$$
(1+\eta) \max \left(\frac{\partial g_{\overline{\mathbf{C} \backslash \gamma_{0}}}(0)}{\partial \mathbf{n}_{+}}, \frac{\partial g_{\overline{\mathbf{C} \backslash \gamma_{0}}}(0)}{\partial \mathbf{n}_{-}}\right) .
$$

On the left in (2.17) we have (see (i))

$$
\left(P_{n} Q_{\varepsilon n}\right)^{\prime}(0)=P_{n}^{\prime}(0)+P_{n}(0) Q_{\varepsilon n}^{\prime}(0),
$$


and for the second term we get again from the Kalmykov-Nagy theorem that (note $\left\|Q_{\varepsilon n}\right\|_{\gamma_{\tau}} \leq 1$ )

$$
\left|P_{n}(0) Q_{\varepsilon n}^{\prime}(0)\right| \leq(1+o(1)) n \varepsilon\left\|P_{n}\right\|_{\gamma_{0}} \max \left(\frac{\partial g_{\overline{\mathbf{C} \backslash \gamma_{\tau}}}(0)}{\partial \mathbf{n}_{+}}, \frac{\partial g_{\overline{\mathbf{C} \backslash \gamma_{\tau}}}(0)}{\partial \mathbf{n}_{-}}\right),
$$

and we can apply again (2.11) to the right hand side.

All in all, we obtain

$$
\left|P_{n}^{\prime}(0)\right| \leq(1+o(1)) n(1+2 \varepsilon)(1+\eta)\left\|P_{n}\right\|_{\gamma_{0}} \max \left(\frac{\partial g_{\overline{\mathbf{C} \backslash \gamma_{0}}}(0)}{\partial \mathbf{n}_{+}}, \frac{\partial g_{\overline{\mathbf{C} \backslash \gamma_{0}}}(0)}{\partial \mathbf{n}_{-}}\right) .
$$

Now this is true for all $\varepsilon, \eta>0$, so the claim in the theorem follows.

In proving the last statement about uniformity some caution has to be made because the estimate (1.3) is not known to be uniform for families of analytic curves.

First of all, by compactness it is enough to prove that for any $\varepsilon, \eta>0$ and for any $z_{0} \in J$ there is a $\delta>0$ such that for sufficiently large $n$ and for all $w \in \Gamma$ with $\left|w-z_{0}\right|<\delta$ we have the following analogue of (2.18):

$$
\left|P_{n}^{\prime}(w)\right| \leq n(1+3 \varepsilon)(1+2 \eta)\left\|P_{n}\right\|_{\gamma_{0}} \max \left(\frac{\partial g_{\overline{\mathbf{C} \backslash \gamma_{0}}}(w)}{\partial \mathbf{n}_{+}}, \frac{\partial g_{\overline{\mathbf{C} \backslash \gamma_{0}}}(w)}{\partial \mathbf{n}_{-}}\right) .
$$

We shall do that for $z_{0}=0$ following the preceding proof, which is clearly sufficient, for other points can be similarly handled.

For $\varepsilon, \eta>0$ select a small $\tau<\min \left(\tau_{\eta}, \tau_{\eta}^{*}\right) / 2$ as before, and let $\delta<\tau$ be a small positive number. For $w \in \gamma_{0},\left|w-z_{0}\right|<\delta$, say for $w=\gamma_{0}\left(t_{w}\right)$, consider the transformation

$$
T_{w}(z)=z \gamma_{0}^{\prime}\left(t_{w}\right) / \gamma_{0}^{\prime}(0)+w .
$$

This maps the Jordan arc $\gamma_{\tau}$ into a Jordan arc $\bar{\gamma}_{\tau}$ which passes through the point $w$ and has the same tangent as $\gamma_{0}$ there. Indeed for $|t|<1 / 2$ we have for $\bar{\gamma}_{\tau}$ the parametrization

$$
\bar{\gamma}_{\tau}(t)=\gamma_{\tau}(t) \gamma_{0}^{\prime}\left(t_{w}\right) / \gamma_{0}^{\prime}(0)+w
$$

hence

$$
\bar{\gamma}_{\tau}(0)=w=\gamma_{0}\left(t_{w}\right), \quad \bar{\gamma}_{\tau}^{\prime}(0)=\gamma_{0}^{\prime}\left(t_{w}\right)
$$

because $\gamma_{\tau}^{\prime}(0)=\gamma_{0}^{\prime}(0)$, and we also have

$$
\left|\bar{\gamma}_{\tau}^{\prime \prime}(t)-\gamma_{0}^{\prime \prime}\left(t+t_{w}\right)\right| \leq 2 \tau
$$

if $\delta$ (and together with it $t_{w}$ ) is sufficiently small. (To get this write

$$
\left|\bar{\gamma}_{\tau}^{\prime \prime}(t)-\gamma_{0}^{\prime \prime}\left(t+t_{w}\right)\right| \leq\left|\bar{\gamma}_{\tau}^{\prime \prime}(t)-\gamma_{\tau}^{\prime \prime}(t)\right|+\left|\gamma_{\tau}^{\prime \prime}(t)-\gamma_{0}^{\prime \prime}(t)\right|+\left|\gamma_{\tau}^{\prime \prime}(t)-\gamma_{0}^{\prime \prime}\left(t+t_{w}\right)\right|
$$


and use that the second term is $\leq \tau$ by the construction of $\gamma_{\tau}$, the third term is small by the $C^{2}$ smoothness of $\gamma_{0}=\gamma$, and the first term is small in view of how $T_{w}$ has been defined.) So we get as in (2.4)

$$
\left|\bar{\gamma}_{\tau}(t)-\gamma_{0}\left(t+t_{w}\right)\right| \leq 2 \tau|t|^{2}, \quad\left|\bar{\gamma}_{\tau}^{\prime}(t)-\gamma_{0}^{\prime}\left(t+t_{w}\right)\right| \leq 2 \tau|t|,
$$

and otherwise the distance in between $\bar{\gamma}_{\tau}$ and $\gamma_{0}$ is smaller than $2 \tau$. Now follow the preceding proof by replacing (2.4) by $(2.20)$, and replacing $\gamma_{\tau}$ everywhere by $\bar{\gamma}_{\tau}$. (2.8) remains true (just replace $\tau$ by $2 \tau$ in the estimate). (2.10) becomes

$g_{\overline{\mathbf{C} \backslash \gamma_{0}}}(z) \leq M_{4} \sqrt{\tau}|z-w|^{2}, \quad z \in \bar{\gamma}_{\tau} \quad$ and $\quad g_{\overline{\mathbf{C} \backslash \bar{\gamma}_{\tau}}}(z) \leq M_{4} \sqrt{\tau}|z-w|^{2}, \quad z \in \gamma_{0}$,

and (2.11) takes the form (c.f. (6.1))

$$
\frac{\partial g_{\overline{\mathbf{C} \backslash \bar{\gamma}_{\tau}}}(0)}{\partial \mathbf{n}_{ \pm}}<(1+\eta) \frac{\partial g_{\overline{\mathbf{C} \backslash \gamma_{0}}}(w)}{\partial \mathbf{n}_{ \pm}}
$$

If we use

$$
\bar{Q}_{\varepsilon n}(z)=Q_{\varepsilon n}\left(T_{w}^{-1}(z)\right)
$$

instead of the fast decreasing polynomials $Q_{\varepsilon n}$, then we can deduce as in (2.16)

$$
\left\|P_{n} \bar{Q}_{\varepsilon n}\right\|_{\bar{\gamma}_{\tau}} \leq(1+2 \eta)\left\|P_{n}\right\|_{\gamma_{0}}
$$

with the same proof.

Now the crucial observation is that $\bar{\gamma}_{\tau}=T_{w}\left(\gamma_{\tau}\right)$ is a dilated/rotated/translated copy of $\gamma_{\tau}$ and under these transformations the origin corresponds to the point $w$. These similarity transformations clearly preserve the validity of the KalmykovNagy estimate (1.3) so we can conclude the analogue of (2.17) for $\bar{\gamma}_{\tau}$ from (1.3) and for $\left.\mid P_{n} \bar{Q}_{\varepsilon n}\right)^{\prime}(w) \mid$ as before - basically we need to apply (1.3) to the same arc $\gamma_{\tau}$ at the same point (namely at the origin), but for a different polynomial, namely for $P_{n}\left(T_{w}(z)\right) Q_{\varepsilon n}(z)$. Now from that analogue of (2.17) we get (2.19) for large $n$ exactly as (2.18) was obtained from (2.17).

This completes the proof of Theorem 1.

Remark 5 For later use we remark the following. The proof depended on the inequality (2.10), which was the consequence of (2.8) and (2.9). (2.8) does not require any smoothness, and to get (2.10) one needs to prove (2.9) for points close to the origin. Using the $C^{2}$ property of $\Gamma$ we have done that for all $z=\gamma_{\tau}(t)$ with $t \in[-1 / 2,1 / 2]$, but the verification of (2.9) for points $z=\gamma_{\tau}(t)$ with $t \in\left[-t_{0}, t_{0}\right]$, with any fixed $0<t_{0}<1$ would just as well suffice. Now if we know the $C^{2}$ property of $\Gamma$ only in a neighborhood of the point $z_{0}=0$, say we know the $C^{2}$ smoothness of $\gamma(t)$ only for $\left[-2 t_{0}, 2 t_{0}\right]$, then define $\gamma_{\tau}(t)$ as in $(2.3)$ for $t \in\left[-2 t_{0}, 2 t_{0}\right]$, and for other values just make sure that $\gamma_{\tau}(t)$ is closer to $\gamma(t)=\gamma_{0}(t)$ than $\tau$. With this modification the proof in Appendix 1 supplies (2.9) close to the origin via the uniform Hölder 1 property of the 
Green's functions $g_{\overline{\mathbf{C} \backslash \gamma_{\tau}}}(z)$ in a neighborhood of the origin, see Remark 8 at the end of Appendix 1. Thus, the proof goes through in this case, as well, so we have

Corollary 6 To make the conclusion in Theorem 1 at a point $z_{0} \in \Gamma$, the $C^{2}$ property of $\Gamma$ is needed only in a neighborhood of $z_{0}$.

\section{Proof of Theorem 4}

We prove the theorem by induction on $k$, the $k=1$ case has been done in Theorem 1.

By repeated use of Pommerenke's theorem [13] we have

$$
\left\|R_{n}^{(k)}\right\|_{\Gamma} \leq C_{k} n^{2 k}\left\|R_{n}\right\|_{\Gamma}
$$

for polynomials $R_{n}$ of degree $\leq n$, where the constant $C_{k}$ depends only on $k$ and $\Gamma$.

Let

$$
M(u)=\max \left(\frac{\partial g_{\overline{\mathbf{C} \backslash \Gamma}}(u)}{\partial \mathbf{n}_{+}}, \frac{\partial g_{\overline{\mathbf{C} \backslash \Gamma}}(u)}{\partial \mathbf{n}_{-}}\right) .
$$

Suppose that the claim is true for a $k$ and for all subarcs $J$ as in the theorem. For such a subarc select a subarc $J \subset J^{*}$ such that $J^{*}$ has no common endpoint either with $J$ or with $\Gamma$. For a $z_{0} \in J$ let $Q(v)=Q_{n^{1 / 3}, z_{0}}(v)$ be the fast decreasing polynomial for $\Gamma$ as in (i)-(iii) of (2.13), i.e. a polynomial of degree at most $n^{1 / 3}$ such that $Q\left(z_{0}\right)=1,\|Q\|_{\Gamma} \leq 1$ and if $v \in \Gamma$, then

$$
|Q(v)| \leq C_{0} e^{-c_{0} n^{1 / 3}\left|v-z_{0}\right|^{2}} .
$$

Since $\Gamma$ is $C^{2}$ smooth, the constants $C_{0}, c_{0}$ here are independent of $z_{0} \in J$.

Consider any $\delta>0$ such that the intersection of $\Gamma$ with the $\delta$-neighborhood of $J$ is part of $J^{*}$, and set $f_{k, n, z_{0}}(v)=P_{n}^{(k)}(v) Q(v)$. On $\Gamma$ for this we have the bound

$$
O\left(n^{2 k}\right) \exp \left(-c_{0} n^{1 / 3} \delta^{2}\right)\left\|P_{n}\right\|_{\Gamma}=o(1)\left\|P_{n}\right\|_{\Gamma}
$$

outside the $\delta$-neighborhood of $z_{0}$ (see (3.1) and (3.2)), while in the $\delta$-neighborhood of any $z_{0} \in J$ we have, by the induction hypothesis applied to $P_{n}$ and to the $\operatorname{arc} J^{*}$,

$$
\begin{aligned}
\left|f_{k, n, z_{0}}(v)\right| & \leq(1+o(1)) n^{k}\left\|P_{n}\right\|_{\Gamma} M(v)^{k} \\
& \leq(1+o(1)) n^{k}(1+\varepsilon)^{k}\left\|P_{n}\right\|_{\Gamma} M\left(z_{0}\right)^{k}
\end{aligned}
$$

where $\varepsilon \rightarrow 0$ as $\delta \rightarrow 0$. Here we used that, by the continuity of $M\left(z_{0}\right)$ (which is a consequence of the continuity of the normal derivatives in its definition which is a consequence of the $C^{2}$ smootness of $\Gamma$ ), if $z_{0} \in J$ and $\left|v-z_{0}\right|<\delta$, then $M(v) \leq(1+\varepsilon) M\left(z_{0}\right)$ with some $\varepsilon$ that tends to 0 as $\delta \rightarrow 0$. Therefore, $f_{k, n, z_{0}}(v)$ is a polynomial in $v$ of degree at most $n+n^{1 / 3}$ for which

$$
\left\|f_{k, n, z_{0}}\right\|_{\Gamma} \leq(1+o(1)) n^{k}\left\|P_{n}\right\|_{\Gamma} M\left(z_{0}\right)^{k}
$$


and upon applying Theorem 1 to the polynomial $f_{k, n, z_{0}}$ we obtain

$$
\left|f_{k, n, z_{0}}^{\prime}\left(z_{0}\right)\right| \leq(1+o(1)) n^{k+1}\left\|P_{n}\right\|_{\Gamma} M\left(z_{0}\right)^{k+1} .
$$

On the left (recall that $Q\left(z_{0}\right)=1$ )

$$
f_{k, n, z_{0}}^{\prime}\left(z_{0}\right)=P_{n}^{(k+1)}\left(z_{0}\right)+P_{n}^{(k)}\left(z_{0}\right) Q^{\prime}\left(z_{0}\right),
$$

and the second term on the right is of order $O\left(n^{2 / 3}\right) O\left(n^{k}\right)\left\|P_{n}\right\|_{\Gamma}$ by (3.1) applied to $Q$ and by the induction assumption. Therefore, from (3.3) we can conclude (1.9) for $k+1$.

From how we derived this, it follows that this estimate is uniform in $z_{0} \in J$.

\section{Proof of Theorem 2}

We shall first prove

$$
\left|P_{n}^{(k)}(A)\right| \leq(1+o(1)) n^{2 k} \frac{2^{k} \Omega(A)^{2 k}}{(2 k-1) ! !}\left\|P_{n}\right\|_{\Gamma} .
$$

We may assume that $A=0$ and that the positive $x$ axis is the half-tangent to $\Gamma$ at 0 .

Let

$$
\Gamma^{*}=\left\{z \mid z^{2} \in \Gamma\right\} .
$$

This is a Jordan arc that is symmetric onto the origin. One can prove (see Appendix 2 for the proof) that $\Gamma^{*}$ has $C^{2}$ smoothness.

Let $P_{n}$ be an arbitrary polynomial of degree at most $n$, and set $R_{2 n}(x)=$ $P_{n}\left(x^{2}\right)$. If we apply Theorem 4 to $\Gamma^{*}$ and to the polynomial $R_{2 n}$, then we get

$$
\left|R_{2 n}^{(2 k)}(0)\right| \leq(1+o(1))(2 n)^{2 k} M^{*}(0)^{2 k}\left\|R_{2 n}\right\|_{\Gamma^{*}},
$$

where $M^{*}(0)$ is the maximum of the two normal derivatives of $g_{\overline{\mathbf{C}} \backslash \Gamma^{*}}$ at 0 . Now we need Faà di Bruno's formula [6] (see also [16, pp. 35-37])

$$
(S(F(z)))^{(2 k)}=\sum_{m_{j}} \frac{(2 k) !}{\prod_{j=1}^{2 k} m_{j} !(j !)^{m_{j}}} S^{\left(m_{1}+\cdots+m_{2 k}\right)}(F(z)) \prod_{j=1}^{2 k}\left(F^{(j)}(z)\right)^{m_{j}},
$$

where the summation is for all nonnegative integers $m_{1}, \ldots, m_{2 k}$ for which $m_{1}+$ $2 m_{2}+3 m_{3}+\cdots+2 k m_{2 k}=2 k$. Apply this with $S(z)=P_{n}(z)$ and $F(z)=z^{2}$ at $z=0$ :

$$
\begin{aligned}
R_{2 n}^{(2 k)}(0) & =\left.\left(P_{n}(F(z))\right)^{(2 k)}\right|_{z=0} \\
& =\sum_{m_{j}} \frac{(2 k) !}{\prod_{j=1}^{2 k} m_{j} !(j !)^{m_{j}}} P_{n}^{\left(m_{1}+\cdots+m_{2 k}\right)}(0) \prod_{j=1}^{2 k}\left(F^{(j)}(0)\right)^{m_{j}} \\
& =\frac{(2 k) !}{k ! 2^{k}} P_{n}^{(k)}(0) 2^{k}
\end{aligned}
$$


(note that $F^{(j)}(0)=0$ unless $j=2$ and $F^{(2)}(0)=2$ ), and so, in view of (4.2),

$$
\left|P_{n}^{(k)}(0)\right| \leq(1+o(1)) \frac{2^{k}}{(2 k-1) ! !} n^{2 k} M^{*}(0)^{2 k}\left\|P_{n}\right\|_{\Gamma},
$$

where we also used that $\left\|R_{2 n}\right\|_{\Gamma^{*}}=\left\|P_{n}\right\|_{\Gamma}$.

Finally, since

$$
g_{\overline{\mathbf{C}} \backslash \Gamma^{*}}(z, \infty)=\frac{1}{2} g_{\overline{\mathbf{C}} \backslash \Gamma}\left(z^{2}, \infty\right),
$$

it follows that

$$
\frac{\partial g_{\overline{\mathbf{C} \backslash \Gamma^{*}}}(z)}{\partial \mathbf{n}_{ \pm}(z)}=\frac{1}{2} \frac{\partial g_{\overline{\mathbf{C} \backslash \Gamma}}\left(z^{2}\right)}{\partial \mathbf{n}_{ \pm}\left(z^{2}\right)}|2 z| .
$$

Hence, since $\Gamma^{*}$ is $C^{2}$ smooth,

$$
\frac{\partial g_{\overline{\mathbf{C} \backslash \Gamma^{*}}}(0)}{\partial \mathbf{n}_{ \pm}}=\lim _{w \rightarrow 0} \frac{\partial g_{\overline{\mathbf{C} \backslash \Gamma}}(w, \infty)}{\partial \mathbf{n}_{ \pm}(w)} \sqrt{|w|} .
$$

By the symmetry of the curve $\Gamma^{*}$ onto the origin we obtain that the two normal derivatives on the left hand side are the same, and we can conclude that the two limits on the right are also the same, and we called the common limit $\Omega_{\Gamma}(0)$ in (1.4). This verifies

$$
M^{*}(0)=\Omega_{\Gamma}(0) .
$$

Therefore, (4.4) proves (4.1).

So far we have verified (4.1), which is the claim in the theorem, but only at the endpoint $A$ of the arc $\Gamma$. We shall reduce the Markov type inequality in the theorem to this special case.

If $z \in \Gamma$ is close to $A=0$, then consider the $\operatorname{arc} \Gamma_{z}$ which is the $\operatorname{arc}$ of $\Gamma$ from $z$ to $B$, so the endpoints of $\Gamma_{z}$ are $B$ and $z$. The preceding proof of (4.1) was uniform in the sense that it holds uniformly for all $\Gamma_{z}, z \in \Gamma,|z-A| \leq|B-A| / 2$ (see the proof of Theorem 1 and Appendix 1), therefore we obtain (replace in (4.1) $A$ by $z$ )

$$
\left|P_{n}^{(k)}(z)\right| \leq(1+o(1)) n^{2 k} \frac{2^{k} \Omega_{\Gamma_{z}}(z)^{2 k}}{(2 k-1) ! !}\left\|P_{n}\right\|_{\Gamma_{z}},
$$

where now the quantity $\Omega_{\Gamma_{z}}(z)$ must be taken with respect to $\Gamma_{z}$, rather than with respect to $\Gamma$. Since on the right

$$
\left\|P_{n}\right\|_{\Gamma_{z}} \leq\left\|P_{n}\right\|_{\Gamma}
$$

all what remains to prove is

$$
\lim _{z \rightarrow A, z \in \Gamma} \Omega_{\Gamma_{z}}(z) \rightarrow \Omega_{\Gamma}(A) .
$$


Indeed, then we obtain from (4.7) (using also that $\Omega_{\Gamma}(A)$ is not 0 , see (4.6) and Remark 7 in Section 6) that for any $\varepsilon>0$

$$
\left|P_{n}^{(k)}(z)\right| \leq(1+\varepsilon) n^{2 k} \frac{2^{k} \Omega_{\Gamma}(z)^{2 k}}{(2 k-1) ! !}\left\|P_{n}\right\|_{\Gamma},
$$

if $z \in \Gamma$ lies sufficiently close to $A$, say $|z-A| \leq \delta$ and $n$ is sufficiently large. On the other hand, Theorem 1 shows that $P_{n}^{(k)}(z)=O\left(n^{k}\right)$ on subsets of $\Gamma$ lying away from the endpoints $A, B$, in particular this is true for $z \in U,|z-A| \geq \delta$. Now this and (4.9) prove the theorem.

In verifying (4.8) let $h(t), t \in[0,1]$ be a $C^{2}$ parametrization of $\Gamma$ so that $h(0)=A=0$ and $h(1)=B$. For $z \in \Gamma$ let $t_{z} \in[0,1]$ be the point for which $h\left(t_{z}\right)=z$. Then $h_{z}(t):=h\left(t_{z}+t\left(1-t_{z}\right)\right)-z, t \in[0,1]$ is a $C^{2}$ parametrization of $\Gamma_{z}-z$ with parameter interval $[0,1]$. Note that one endpoint of the $\operatorname{arc} \Gamma_{z}-z$ is again at 0 .

Symmetrize $\Gamma_{z}-z$ as $\Gamma$ above, i.e. let

$$
\Gamma_{z}^{*}=\left\{w \mid w^{2} \in \Gamma_{z}-z\right\} .
$$

In Appendix 2 it is proven (see also Remark 9) that in a neighborhood of the origin $\Gamma_{z}^{*}$ has a $C^{2}$ parametrization $h_{z}^{*}(t)$ that can be written explicitly in terms of $h_{z}(t)$, and then it easily follows that $h_{z}^{*}(t) \rightarrow h_{0}^{*}(t),\left(h_{z}^{*}\right)^{\prime}(t) \rightarrow\left(h_{0}^{*}\right)^{\prime}(t)$ and $\left(h_{z}^{*}\right)^{\prime \prime}(t) \rightarrow\left(h_{0}^{*}\right)^{\prime \prime}(t)$ uniformly in $t \in\left[-t_{0}, t_{0}\right]$ for some $t_{0}>0$ as $z \rightarrow 0(z \in \Gamma)$. But then Appendix 1 (see particularly (6.1) and Remark 8) gives

$$
\frac{\partial g_{\overline{\mathbf{C} \backslash \Gamma_{z}^{*}}}(0)}{\partial \mathbf{n}_{ \pm}} \rightarrow \frac{\partial g_{\overline{\mathbf{C} \backslash \Gamma^{*}}}(0)}{\partial \mathbf{n}_{ \pm}}
$$

as $z \rightarrow 0(z \in \Gamma)$. Since (see (4.6))

$$
\frac{\partial g_{\overline{\mathbf{C} \backslash \Gamma^{*}}}(0)}{\partial \mathbf{n}_{ \pm}}=\Omega_{\Gamma_{z}-z}(0)=\Omega_{\Gamma_{z}}(z)
$$

and

$$
\frac{\partial g_{\overline{\mathbf{C} \backslash \Gamma^{*}}}(0)}{\partial \mathbf{n}_{ \pm}}=\Omega_{\Gamma}(0),
$$

(4.8) follows from (4.10), and with it the proof of Theorem 2 is complete.

\section{$5 \quad$ Proof of Theorem 3}

The proof is along the lines of the first part of Theorem 2. Consider again the symmetric arc

$$
\Gamma^{*}=\left\{z \mid z^{2} \in \Gamma\right\} .
$$

The optimality of the best Bernstein factor for smooth Jordan curves in the paper [12, Theorem 3] (see also [11, Theorem 2] and its proof) can be extended 
to higher derivatives with the same proof, hence, it follows that there are polynomials $R_{2 n}$ of degree $n=2,4, \ldots$ such that

$$
\left|R_{2 n}^{(2 k)}(0)\right| \geq(1+o(1))(2 n)^{2 k} M^{*}(0)^{2 k}\left\|R_{2 n}\right\|_{\Gamma^{*}},
$$

where $M^{*}(0)$ is the normal derivative of $g_{\overline{\mathbf{C}} \backslash \Gamma^{*}}$ at 0 from either side (the two normal derivatives are the same because of the symmetry of $\left.\Gamma^{*}\right)$. Since $\frac{1}{2}\left(R_{2 n}(z)+\right.$ $R_{2 n}(-z)$ ) also has this property, we may assume that $R_{2 n}$ is even, and then set $P_{n}\left(z^{2}\right)=R_{2 n}(z)$. Apply again Faà di Bruno's formula (4.3) with $F(z)=z^{2}$ at $z=0$ to conclude as before that

$$
R_{2 n}^{(2 k)}(0)=\frac{(2 k) !}{k ! 2^{k}} P_{n}^{(k)}(0) 2^{k},
$$

and so

$$
\left|P_{n}^{(k)}(0)\right| \geq(1+o(1)) \frac{1}{2^{k}(2 k-1) ! !}(2 n)^{2 k} M^{*}(0)^{2 k}\left\|P_{n}\right\|_{\Gamma} .
$$

Now Theorem 3 follows from here and from (4.6).

\section{Appendix 1}

In this appendix we prove that the Green's functions $g_{\overline{\mathbf{C}} \backslash \gamma_{\tau}}, 0 \leq \tau \leq \tau_{0}$, in the proof of Theorem 1 (see Section 2) are uniformly Hölder 1 continuous in a neighborhood of the origin which contains the $\operatorname{arcs}\left\{\gamma_{\tau}(t) \mid t \in[-1 / 2,1 / 2]\right\}$. We shall also prove a convergence theorem for the normal derivative of these Green's functions (see (6.1)).

It is enough to prove the Hölder 1 property separately on the two sides of $\gamma_{\tau}$, so we may concentrate on the "left" side corresponding to the orientation of $\gamma_{\tau}$ (matching increasing parameter values). Consider the polynomial $S=S_{\tau}$ on the interval $[3 / 4,1]$ of degree at most 5 for which

$S(3 / 4)=\left(\gamma_{\tau}-\gamma\right)(3 / 4), \quad S^{\prime}(3 / 4)=\left(\gamma_{\tau}-\gamma\right)^{\prime}(3 / 4), \quad S^{\prime \prime}(3 / 4)=\left(\gamma_{\tau}-\gamma\right)^{\prime \prime}(3 / 4)$,

and

$$
S(1)=0, \quad S^{\prime}(1)=0, \quad S^{\prime \prime}(1)=0 .
$$

Then $\gamma+S_{\tau}$ has the same 0th, 1st and 2nd derivatives as $\gamma_{\tau}$ at the point $3 / 4$ and the corresponding derivatives match those of $\gamma$ at 1 . In a similar manner, let $R=R_{\tau}$ be the polynomial of degree 5 satisfying

$$
\begin{gathered}
R(-3 / 4)=\left(\gamma_{\tau}-\gamma\right)(-3 / 4), \quad R^{\prime}(3 / 4)=\left(\gamma_{\tau}-\gamma\right)^{\prime}(-3 / 4), \\
R^{\prime \prime}(-3 / 4)=\left(\gamma_{\tau}-\gamma\right)^{\prime \prime}(-3 / 4),
\end{gathered}
$$

and

$$
R(-1)=0, \quad R^{\prime}(-1)=0, \quad R^{\prime \prime}(-1)=0 .
$$




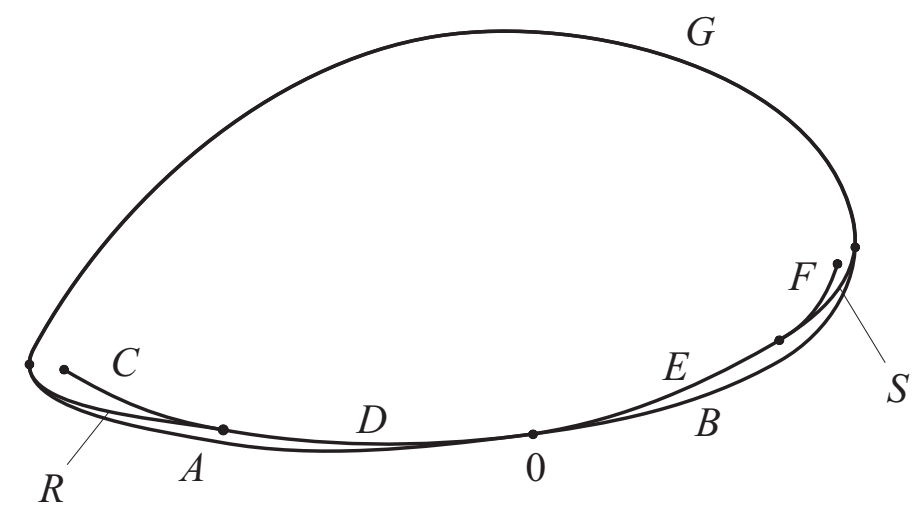

Figure 1: A schematic figure of the original arc $\gamma$ (consisting of the pieces $A, B$ ), of $\gamma_{\tau}$ (consisting of $C, D, E, F$ ), of the interpolating $\operatorname{arcs} S$ and $R$, as well as the common connecting arc $G$ that completes $\gamma$ and $\tilde{\gamma}_{\tau}$ to a Jordan curve. In this figure $\tilde{\gamma}_{\tau}$ consists of $R, D, E, S$.

If we set

$$
\tilde{\gamma}_{\tau}(t)= \begin{cases}\gamma(t)+R(t), & t \in[-1,-3 / 4] \\ \gamma_{\tau}(t), & t \in[-3 / 4,3 / 4] \\ \gamma(t)+S(t), & t \in[3 / 4,1],\end{cases}
$$

then $\tilde{\gamma}_{\tau}$ is twice continuously differentiable on $[-1,1]$, it coincides with $\gamma_{\tau}$ on $[-3 / 4,3 / 4]$, and it has the same derivatives up to order 2 as $\gamma$ both at 1 and at -1 . Extend $\gamma$ to a $C^{2}$ (closed) Jordan curve $\gamma^{*}, t \in[-1,2]$, by attaching a $\gamma(t)$, $t \in[1,2]$ to the original $\gamma$ which joins its endpoints $\gamma( \pm 1)$ (see Figure 1.). If we extend each $\tilde{\gamma}_{\tau}$ to a $\gamma_{\tau}^{*}$ by the same $\gamma(t), t \in[1,2]$, i.e. if we set

$$
\gamma_{\tau}^{*}(t)= \begin{cases}\tilde{\gamma}_{\tau}(t), & t \in[-1,1], \\ \gamma(t), & t \in[1,2],\end{cases}
$$

then $\gamma_{\tau}^{*}$ are (closed) $C^{2}$ Jordan curves such that $\gamma_{\tau}^{*} \rightarrow \gamma^{*},\left(\gamma_{\tau}^{*}\right)^{\prime} \rightarrow\left(\gamma^{*}\right)^{\prime}$, and $\left(\gamma_{\tau}^{*}\right)^{\prime \prime} \rightarrow\left(\gamma^{*}\right)^{\prime \prime}$ as $\tau \rightarrow 0$ (this follows from the fact that, by the assumption on $\gamma_{\tau}$ and $\gamma$, the polynomials $S, R$ clearly satisfy $\left|S_{\tau}(t)\right| \leq C_{1} \tau, t \in[3 / 4,1]$ and $\left|R_{\tau}(t)\right| \leq C_{1} \tau, t \in[-1,-3 / 4]$, along with similar estimates on their first and second derivatives). We denote by $G_{\tau}^{*}$ the inner domain to $\gamma_{\tau}^{*}$. If we do the extension $\gamma \rightarrow \gamma^{*}$ properly, then $G_{\tau}^{*}$ contains a left neighborhood of $\gamma_{\tau}$.

Let $w_{0} \in G_{\tau}^{*}$ be a fixed point inside all $\gamma_{\tau}^{*}$ (e.g. we can set $w_{0}=i y_{0}$ for some small $y_{0}>0$ ). Clearly, there is a $d$ such that the disk of radius $d$ about $w_{0}$ lies in all $G_{\tau}^{*}, 0 \leq \tau \leq \tau_{0}$, and the diameter of all the $\gamma_{\tau}^{*}$ is at most $1 / d$. Let $\varphi_{\tau}$ be the conformal map from the unit disk $\Delta_{1}$ onto $G_{\tau}^{*}$ such that $\varphi_{\tau}(0)=w_{0}$ and $\varphi_{\tau}(1)=0$. By Theorem III* and Theorem IV of [21] the functions $\varphi_{\tau}$ and $\varphi_{\tau}^{\prime}$ can be extended continuously to the closed unit disk $\bar{\Delta}_{1}$, the extensions are uniformly Hölder $1 / 2$ on $\bar{\Delta}_{1}$, and we have $1 / \Lambda \leq\left|\varphi_{\tau}^{\prime}(z)\right| \leq \Lambda, z \in \bar{\Delta}_{1}$, with a constant $\Lambda$ that is independent of $\tau \leq \tau_{0}$ (the constant $\Lambda$ depends only on 
$d$ above and on the bounds for $\gamma^{\prime}, 1 / \gamma^{\prime}$ and $\gamma^{\prime \prime}$, which furnish uniform bounds for $\left(\gamma_{\tau}^{*}\right)^{\prime},\left(\gamma_{\tau}^{*}\right)^{\prime}$ and $\left(\gamma_{\tau}^{*}\right)^{\prime \prime}$ by our construction). Furthermore, as $\tau \rightarrow 0$ we have $\varphi_{\tau}(z) \rightarrow \varphi_{0}(z), \varphi_{\tau}^{\prime}(z) \rightarrow \varphi_{0}^{\prime}(z)$ uniformly in $z \in \bar{\Delta}_{1}$.

The arc $\left\{\gamma_{\tau}(t) \mid t \in[-3 / 4,3 / 4]\right\}$ is mapped by $\varphi_{\tau}^{-1}$ onto a subarc $J_{\tau}$ of the unit circle that contains the point 1 , and, as $\tau \rightarrow 0$, these arcs tend to the corresponding arc $J_{0}$ with respect to $\varphi_{0}^{-1}$. Let $I$ be a proper closed subarc of the interior of $J_{0}$. We may assume $I$ so large that for sufficiently small $\tau$ the arc $I$ contains the $\operatorname{arcs}\left\{\varphi_{\tau}^{-1}\left(\gamma_{\tau}(t)\right) \mid t \in[-5 / 8,5 / 8]\right\}$. Without loss of generality we may assume that this holds for all $\tau \leq \tau_{0}$.

Next, note that, by (2.8) and the maximum principle (for $\pm\left(g_{\overline{\mathbf{C}} \backslash \gamma_{\tau}}-g_{\overline{\mathbf{C}} \backslash \gamma}\right)$ ), the function $g_{\overline{\mathbf{C} \backslash \gamma_{\tau}}}(z)-g_{\overline{\mathbf{C} \backslash \gamma}}(z)$ tends uniformly to 0 on the whole complex plane as $\tau \rightarrow 0$. Now consider $h_{\tau}(z)=g_{\overline{\mathbf{C}} \backslash \gamma_{\tau}}\left(\varphi_{\tau}(z)\right)$. These are harmonic functions on the unit disk that vanish on the arcs $J_{\tau}$ and converge uniformly on the closed unit disk to $h_{0}(z)$ as $\tau \rightarrow 0$. Since $I$ lies of positive distance (independent of $\tau$ ) from $\partial \Delta_{1} \backslash J_{\tau}$, Poisson's formula (see (6.2) below) implies that the functions $h_{\tau}(z)$ are uniformly Hölder 1 continuous on compact subsets of the open unit disk, as well as on the set $\tilde{I}=\left\{r e^{i \theta} \mid 0 \leq r \leq 1, e^{i \theta} \in I\right\}$. But then $g_{\overline{\mathbf{C}} \backslash \gamma_{\tau}}(z)=h_{\tau}\left(\varphi_{\tau}^{-1}(z)\right)$ are uniformly Hölder 1 continuous on the sets $\varphi_{\tau}(\tilde{I})$ because

$$
\left|\left(\varphi_{\tau}^{-1}\right)^{\prime}(z)\right|=\frac{1}{\left|\varphi_{\tau}^{\prime}\left(\varphi_{\tau}^{-1}(z)\right)\right|}
$$

are uniformly bounded on their domain. But this last set $\varphi_{\tau}(\tilde{I})$ contains a left neighborhood of the arc $\gamma_{\tau}(t), t \in[-1 / 2,1 / 2]$, which proves the claim that $g_{\overline{\mathbf{C}} \backslash \gamma_{\tau}}$ are uniformly Hölder 1 continuous on a neighborhood of the origin which contains the $\operatorname{arcs} \gamma_{\tau}(t), t \in[-1 / 2,1 / 2]$.

It also follows that, as $\tau \rightarrow 0$, we have

$$
\frac{\partial g_{\overline{\mathbf{C} \backslash \gamma_{\tau}}}\left(\gamma_{\tau}(t)\right)}{\partial \mathbf{n}\left(\gamma_{\tau}(t)\right)} \rightarrow \frac{\partial g_{\overline{\mathbf{C} \backslash \gamma}}(\gamma(t))}{\partial \mathbf{n}(\gamma(t))}
$$

uniformly for $t \in[-1 / 2,1 / 2]$, where $\mathbf{n}\left(\gamma_{\tau}(t)\right)$ denotes the (left) normal to $\gamma_{\tau}$ at the point $\gamma_{\tau}(t)$. To prove this note that, by Poisson's formula,

$$
h_{\tau}\left(r e^{i \theta}\right)=\frac{1}{2 \pi} \int_{-\pi}^{\pi} h_{\tau}\left(e^{i t}\right) \frac{1-r^{2}}{1-2 r \cos (t-\theta)+r^{2}} d t .
$$

Hence, if $\mathbf{n}_{e^{i \theta}}$ denotes the inner normal to the unit circle at the point $e^{i \theta}$, then for $e^{i \theta} \in I$ (which implies $h\left(e^{i \theta}\right)=0$ )

$$
\begin{aligned}
\frac{\partial h_{\tau}\left(e^{i \theta}\right)}{\partial \mathbf{n}_{e^{i \theta}}} & =\lim _{r \nearrow 1} \frac{1+r}{2 \pi} \int_{-\pi}^{\pi} h_{\tau}\left(e^{i t}\right) \frac{1}{1-2 r \cos (t-\theta)+r^{2}} d t \\
& =\frac{1}{\pi} \int_{-\pi}^{\pi} h_{\tau}\left(e^{i t}\right) \frac{1}{4 \sin ^{2}((t-\theta) / 2)} d t
\end{aligned}
$$


so this normal derivative is uniformly continuous and positive on $I$ (recall that $h_{\tau}$ vanishes on a fixed larger arc), furthermore uniformly on $I$

$$
\frac{\partial h_{\tau}\left(e^{i \theta}\right)}{\partial \mathbf{n}_{e^{i \theta}}} \rightarrow \frac{\partial h_{0}\left(e^{i \theta}\right)}{\partial \mathbf{n}_{e^{i \theta}}}
$$

as $\tau \rightarrow 0$ because $h_{\tau} \rightarrow h_{0}$ uniformly on $\overline{\Delta_{1}}$. But if $\xi \in I$ and $\varphi_{\tau}(\xi)=\gamma_{\tau}(t)$, then

$$
\frac{\partial g_{\overline{\mathbf{C}} \backslash \gamma_{\tau}}\left(\gamma_{\tau}(t)\right)}{\partial \mathbf{n}\left(\gamma_{\tau}(t)\right)}=\frac{\partial h_{\tau}(\xi)}{\partial \mathbf{n}_{\xi}}\left|\left(\varphi_{\tau}^{-1}\right)^{\prime}\left(\gamma_{\tau}(t)\right)\right|=\frac{\partial h_{\tau}(\xi)}{\partial \mathbf{n}_{\xi}} \frac{1}{\left|\varphi_{\tau}^{\prime}(\xi)\right|},
$$

so (6.1) is a consequence of (6.4) and of the uniform convergence of $\varphi_{\tau}^{\prime}$ to $\varphi_{0}^{\prime}$ (recall also that for $t \in[-1 / 2,1 / 2]$ we have $\varphi_{\tau}^{-1}\left(\gamma_{\tau}(t)\right) \in I$ ).

Remark 7 The normal derivatives in (6.1) are uniformly bounded from below and above (just use (6.3)).

Remark 8 If we know the $C^{2}$ property of $\Gamma$ (and hence those of $\gamma_{\tau}$ ) only in a neighborhood of $z_{0}=0$, then the preceding proof gives the Hölder 1 property in a neighborhood of $z_{0}=0$. Indeed, if the parametrization $\gamma$ of $\Gamma$ is $C^{2}$ continuous on an interval $\left[-t_{0}, t_{0}\right]$, then just carry out the preceding proof with $\bar{\gamma}(t)=\gamma\left(t / t_{0}\right)$ but still using the function $g_{\overline{\mathbf{C}} \backslash \gamma_{\tau}}$ rather than $g_{\overline{\mathbf{C}} \backslash \bar{\gamma}_{\tau}}$.

The same applies to (6.1), namely to prove it, say, for $t \in\left[-t_{0} / 2, t_{0} / 2\right]$, one only needs to assume $\gamma_{\tau}(t) \rightarrow \gamma(t), \gamma_{\tau}^{\prime}(t) \rightarrow \gamma^{\prime}(t)$ and $\gamma_{\tau}^{\prime \prime}(t) \rightarrow \gamma^{\prime \prime}(t)$ only for $t \in\left[-t_{0}, t_{0}\right]$ (besides the global condition that $\gamma_{\tau}$ lies close to $\gamma$ ).

\section{Appendix 2}

In this appendix we prove that if $\gamma$ is a $C^{2}$ Jordan arc with one endpoint at the origin and

$$
\gamma^{*}=\left\{z \mid z^{2} \in \gamma\right\}
$$

then $\gamma^{*}$ is again $C^{2}$ smooth.

Since the $C^{2}$ smoothness of $\gamma^{*}$ is clear away from the origin, in what follows we shall concentrate on its $C^{2}$ smoothness in a neighborhood of the origin.

Without loss of generality we may assume that the positive half-line is tangent to $\gamma$. The $C^{2}$ smoothness of $\gamma$ means that $\gamma$ has a parametrization $\gamma(t)=(x(t), y(t)), t \in[0,1], \gamma(0)=(0,0)$, such that $x, y$ are two times continuously differentiable, and $\left(x^{\prime}\right)^{2}+\left(y^{\prime}\right)^{2} \neq 0$. By the assumption $y^{\prime}(0)=0$, and we may assume by a linear change of variables that $x^{\prime}(0)=1$. Then $y^{\prime}(t)=O(t)$, $y(t)=O\left(t^{2}\right), x(t) \sim t$, and so $y(t) / x(t) \rightarrow 0$ as $t \rightarrow 0$. (Here, and in what follows, $A \sim B$ means that $A / B$ and $B / A$ are uniformly bounded.)

We change to polar coordinates in a neighborhood of the origin:

$$
r=f(t)=\sqrt{x(t)^{2}+y(t)^{2}}, \quad \varphi=g(t)=\arctan (y(t) / x(t)) .
$$


Then, according to what just has been mentioned, $x(t) / r(t) \rightarrow 1$. Next,

$$
\frac{d r}{d t}=f^{\prime}(t)=\frac{1}{\sqrt{x(t)^{2}+y(t)^{2}}}\left(x(t) x^{\prime}(t)+y(t) y^{\prime}(t)\right),
$$

which is positive on an interval $(0, a]$ (its limit at 0 is 1 ).

The following reasoning will be restricted to this interval $(0, a]$. Thus, $r$, as a function of $t$, is strictly increasing, and we can consider its inverse: $t=f^{-1}(r)$. Then in the polar form $\gamma(t)=r e^{i \varphi}$ we can consider $\varphi$ as a function of $r$ : $\varphi(r)=g\left(f^{-1}(r)\right)$, and we shall first understand what the $C^{2}$ property of $\gamma$ means in terms of this $\varphi(r)$.

From (7.1) it is clear that $d r / d t$ is continuous (as a function of $t$ ), hence $d t / d r$ is also continuous as a function of $r$. Next,

$$
\begin{aligned}
\frac{d^{2} r}{d t^{2}}=f^{\prime \prime}(t) & =-\frac{1}{\sqrt{x(t)^{2}+y(t)^{2}}}\left(x(t) x^{\prime}(t)+y(t) y^{\prime}(t)\right)^{2} \\
& +\frac{1}{\sqrt{x(t)^{2}+y(t)^{2}}}\left(x^{\prime}(t)^{2}+y^{\prime}(t)^{2}+x(t) x^{\prime \prime}(t)+y(t) y^{\prime \prime}(t)\right) .
\end{aligned}
$$

This is continuous on $(0, a]$, and we show that it has a limit at 0 . Using that $x(t) \sim t, y(t) \sim t^{2}, y^{\prime}(t) \sim t$, it is immediate that, upon expansion, all terms have limit, except for the terms

$$
-\frac{1}{{\sqrt{x(t)^{2}+y(t)^{2}}}^{3}}\left(x(t) x^{\prime}(t)\right)^{2}+\frac{1}{\sqrt{x(t)^{2}+y(t)^{2}}} x^{\prime}(t)^{2} .
$$

But this expression is

$$
\frac{1}{{\sqrt{x(t)^{2}+y(t)^{2}}}^{3}}\left(y(t) x^{\prime}(t)\right)^{2},
$$

hence it has 0 limit at 0 . Thus, we obtained that $d^{2} r / d t^{2}=f^{\prime \prime}(t)$ is continuous on $[0, a]$. Now $d t / d r=d\left(f^{-1}(r)\right) / d r=1 / f^{\prime}\left(f^{-1}(r)\right)$ is continuous on $[0, r(a)]$, and so is

$$
\frac{d^{2} t}{d r^{2}}=\frac{d^{2} f^{-1}(r)}{d r^{2}}=\frac{d\left(1 / f^{\prime}\left(f^{-1}(r)\right)\right)}{d r}=-\frac{f^{\prime \prime}\left(f^{-1}(r)\right)}{f^{\prime}\left(f^{-1}(r)\right)^{3}} .
$$

Now we consider the argument function $g$. For it we have

$$
\frac{d g(t)}{d t}=\frac{y^{\prime}(t) x(t)-x^{\prime}(t) y(t)}{x(t)^{2}+y(t)^{2}} .
$$

This is a continuous function even at the origin (note that $y^{\prime}(t) / x(t)=\left(y^{\prime}(t) / t\right) /(x(t) / t)$ has the limit $y^{\prime \prime}(0)$, and $y(t) / x(t)^{2}$ has the limit $y^{\prime \prime}(0) / 2$ because $\left.x(t) / t \rightarrow 1\right)$.

Next,

$$
\frac{d^{2} g(t)}{d t^{2}}=-\frac{\left(y^{\prime}(t) x(t)-x^{\prime}(t) y(t)\right)\left(2 x(t) x^{\prime}(t)+2 y(t) y^{\prime}(t)\right)}{\left(x(t)^{2}+y(t)^{2}\right)^{2}}+\frac{y^{\prime \prime}(t) x(t)-x^{\prime \prime}(t) y(t)}{x(t)^{2}+y(t)^{2}} .
$$


This may not be continuous any more at 0 , but if we multiply it with $r=$ $\sqrt{x(t)^{2}+y(t)^{2}}$, then it becomes continuous on $[0, a]$. Therefore, $r\left(d^{2} g(t) / d t^{2}\right)$ is continuous on $[0, a]$.

Now

$$
\frac{d^{2} \varphi}{d r^{2}}=\frac{d^{2} g}{d r^{2}}=\frac{d^{2} g}{d t^{2}}\left(\frac{d t}{d r}\right)^{2}+\frac{d g}{d t} \frac{d^{2} t}{d r^{2}}
$$

and what we have obtained so far show that $r\left(d^{2} \varphi / d r^{2}\right)$ is continuous on $[0, r(a)]$.

Let us summarize: the $C^{2}$ property of $\gamma$ around the origin implies that in its polar representation $r e^{i \varphi(r)}$ the function $\varphi$ has the properties that $\varphi, \varphi^{\prime}$ and $r \varphi^{\prime \prime}(r)$ are continuous on some interval $[0, b], r \varphi^{\prime \prime}(r)$ has zero limit at 0 , and $\varphi(0)=0, \varphi^{\prime}(0)=0$. Indeed, all these have been shown in the preceding reasoning except that $r \varphi^{\prime \prime}(r)$ has zero 0 limit at 0 . But that must be the case, for we know that $r \varphi^{\prime \prime}(r)$ has a limit at 0 and if that limit was not 0 , then $\varphi^{\prime}$ would not be continuous at 0 .

Conversely, suppose these properties are true for a function $\varphi(r)$. Consider $r$ as a parameter, and the curve $\gamma$ represented by $r e^{i \varphi(r)}$. This has the parametrization $(x(r), y(r))$ with $r \in[0, b]$ (for some $b), x(r)=r \cos \varphi(r)$, $y(r)=r \sin \varphi(r)$. Then

$$
\frac{d^{2} x(r)}{d r^{2}}=-2 \varphi^{\prime}(r) \sin \varphi(r)-r \varphi^{\prime}(r)^{2} \cos \varphi(r)-r \varphi^{\prime \prime}(r) \sin \varphi(r)
$$

and a very similar expression holds for $d^{2} y(r) / d r^{2}$ (exchange sin and cos and change some of the signs). These forms show that the curve $\gamma$ is $C^{2}$ smooth around the origin by the assumed properties of $\varphi$.

Finally, consider the pre-image $\gamma^{*}$ of $\gamma$ under the mapping $z \rightarrow z^{2}$. Around 0 it has the parametrization $u e^{i \varphi\left(u^{2}\right) / 2}=(\tilde{x}(u), \tilde{y}(u)), \tilde{x}(u)=u \cos \left(\varphi\left(u^{2}\right) / 2\right)$, $\tilde{y}(u)=u \sin \left(\varphi\left(u^{2}\right) / 2\right),-\sqrt{b}<u<\sqrt{b}$, and we want to show that this is $C^{2}$ smooth around 0 . The point 0 divides $\gamma^{*}$ into a "right" and a "left" part that are symmetric onto the origin. Now the parametric representation is $r e^{i \Phi(r)}=$ $(X(r), Y(r))$ for the "right" part and $r e^{i \Phi(r)+i \pi}=-r e^{i \Phi(r)}=-(X(r), Y(r))$ for the "left" part with $\Phi(r)=\varphi\left(r^{2}\right) / 2$, and we claim first that $\Phi, \Phi^{\prime}$ and $r \Phi^{\prime \prime}(r)$ are continuous on some interval $[0, B]$ with zero values at the origin. Since $\Phi^{\prime}(r)=r \varphi^{\prime}\left(r^{2}\right), r \Phi^{\prime \prime}(r)=r \varphi^{\prime}\left(r^{2}\right)+2 r^{2} \varphi^{\prime \prime}\left(r^{2}\right)$, these properties immediately follow from those of $\varphi$. Now the $C^{2}$ continuity of $(\tilde{x}(u), \tilde{y}(u))$ follows: the continuity away from the origin is clear, and at 0 it follows from formula (7.2) (more precisely from its -variant where $\Phi$ replaces $\varphi$ ), since the limit of both $\frac{d^{2} \tilde{x}(r)}{d r^{2}}$ and $\frac{d^{2} \tilde{y}(r)}{d r^{2}}$ is 0 at 0 (from the right, which implies the same from the left by symmetry).

Remark 9 The parametrization $u e^{i \varphi\left(u^{2}\right) / 2}=(\tilde{x}(u), \tilde{y}(u)), \tilde{x}(u)=u \cos \left(\varphi\left(u^{2}\right) / 2\right)$, $\tilde{y}(u)=u \sin \left(\varphi\left(u^{2}\right) / 2\right),-\sqrt{b}<u<\sqrt{b}$, given above for $\gamma^{*}$ in a neighborhood of the origin can be explicitly expressed in terms of the original $C^{2}$ parametrization $(x(t), y(t))$ of $\gamma$. In particular, if $\gamma_{n}$ with parametrization $\left(x_{n}(t), y_{n}(t)\right)$ 
tends to $\gamma$ in the sense that $\left(x_{n}(t), y_{n}(t)\right) \rightarrow(x(t), y(t)),\left(x_{n}(t)^{\prime}, y_{n}(t)^{\prime}\right) \rightarrow$ $\left(x(t)^{\prime}, y(t)^{\prime}\right)$ and $\left(x_{n}^{\prime \prime}(t), y_{n}^{\prime \prime}(t)\right) \rightarrow\left(x^{\prime \prime}(t), y^{\prime \prime}(t)\right)$ in a neighborhood of the origin, then for the corresponding $\gamma_{n}^{*}$ with parametrization $\left(\tilde{x}_{n}(u), \tilde{y}_{n}(u)\right)$ we also have $\left(\tilde{x}_{n}(t), \tilde{y}_{n}(t)\right) \rightarrow(\tilde{x}(t), \tilde{y}(t)),\left(\tilde{x}_{n}(t)^{\prime}, \tilde{y}_{n}(t)^{\prime}\right) \rightarrow\left(\tilde{x}(t)^{\prime}, \tilde{y}(t)^{\prime}\right)$ and $\left(\tilde{x}_{n}^{\prime \prime}(t), \tilde{y}_{n}^{\prime \prime}(t)\right) \rightarrow$ $\left(\tilde{x}^{\prime \prime}(t), \tilde{y}^{\prime \prime}(t)\right)$ in a (possibly smaller) neighborhood of the origin.

The author is grateful to B. Nagy and S. I. Kalmykov for valuable suggestions.

\section{References}

[1] D. H. Armitage and S. J. Gardiner, Classical Potential Theory, Springer Verlag, Berlin, Heidelberg, New York, London, Sidney, Toronto, 2002

[2] S. N. Bernstein, On the best approximation of continuos functions by polynomials of given degree, (O nailuchshem problizhenii nepreryvnykh funktsii posredstrvom mnogochlenov dannoi stepeni), Sobraniye sochinenii, Vol. I, 11-104 (1912), Izd. Akad. Nauk SSSR, Vol. I (1952), Vol. II (1954).

[3] P. Borwein and T. Erdélyi, Polynomials and polynomial inequalities, Graduate Texts in Mathematics, 161, Springer Verlag, New York, 1995.

[4] R. A. DeVore and G. G. Lorentz, Constructive Approximation, Grundlehren der mathematischen Wissenschaften, 303, Springer-Verlag, Berlin, Heidelberg, New York 1993.

[5] A. Eremenko, A Markov-type inequality for arbitrary plane continua, Proc. Amer. Math. Soc., 135(2007), 1505-1510.

[6] C. F. Faà di Bruno, Note sur une nouvelle formule de calcul differentiel, Quarterly J. Pure Appl. Math., 1(1857), 359-360.

[7] S. I. Kalmykov and B. Nagy, Polynomial and rational inequalities on analytic Jordan arcs and domains, J. Math. Anal. Appl., 430(2015), 874-894.

[8] A. A. Markov, Sur une question posée par D.I. Mendeleieff, Izv. Akad. Nauk St. Petersburg, 62(1889), 1-24.

[9] V.A. Markov, On Functions of least deviation from zero in a given interval, (1892), (Russian). Appeared in German with a foreword by Sergei Bernstein as "V. A. Markov, Über Polynome, die in einem gegebenen Intervalle möglichst wenig von Null abweichen", Math. Ann., 77(1916), 213-258.

[10] G. V. Milovanovic, D. S. Mitrinovic and Th. M. Rassias, Topics in Polynomials: Extremal Problems, Inequalities, Zeros, World Scientific Publishing Co., Inc., River Edge, NJ, 1994. 
[11] B. Nagy and V. Totik, Bernstein's inequality for algebraic polynomials on circular arcs, Constr. Approx., 37(2013), 223-232.

[12] B. Nagy and V. Totik, Riesz-type inequalities on general sets, J. Math. Anal. Appl. 416(2014), 344-351.

[13] Ch. Pommerenke, On the derivative of a polynomial, Michigan Math. J., 6(1959), 373-375.

[14] Ch. Pommerenke, Boundary Behavior of Conformal Mappings, Grundlehren der mathematischen Wissenschaften, 299, Springer Verlag, Berlin, Heidelberg New York, 1992.

[15] T. Ransford, Potential Theory in the Complex plane, Cambridge University Press, Cambridge, 1995.

[16] J. Riordan, An introduction to combinatorial analysis, Wiley, New York, 1958.

[17] V. Totik, Christoffel functions on curves and domains, Trans. Amer. Math. Soc., 362(2010), 2053-2087.

[18] M. Tsuji, Potential Theory in Modern Function Theory, Maruzen, Tokyo, 1959

[19] V. S. Videnskii, Extremal estimates for the derivative of a trigonometric polynomial on an interval shorter than its period, Soviet Math. Dokl., 1 (1960), 58.

[20] J. L. Walsh, Interpolation and Approximation by Rational Functions in the Complex Domain, third edition, Amer. Math. Soc. Colloquium Publications, XX, Amer. Math. Soc., Providence, 1960.

[21] S. E. Warschawski, On the higher derivative at the boundary of conformal mapping, Trans. Amer. Math. Soc., 38(1935), 310-340.

Bolyai Institute

MTA-SZTE Analysis and Stochastics Research Group

Szeged

Aradi v. tere 1, 6720, Hungary

and

Department of Mathematics and Statistics

University of South Florida

4202 E. Fowler Ave, CMC342

Tampa, FL 33620-5700, USA

totik@mail.usf.edu 